\title{
EDITORIAL
}

\section{Outbreak of Middle East Respiratory Syndrome (MERS): A Public Health Concern}

\author{
Ahmed Raihan SHARIF \\ Medical Officer, Department of Virology, Institute of Epidemiology, Disease Control \& Research, Mohakhali, Dhaka-1212, \\ Bangladesh; Email: drraihan@ gmail.com; Cell no.: +8801715115566
}

Middle East Respiratory Syndrome (MERS) is an illness caused by a virus called Middle East Respiratory Syndrome Coronavirus (MERS-CoV). It is a coronavirus. When referring to the virus and not the illness, CDC uses this acronym. When referring to the illness, CDC uses MERS. It is different from any other coronaviruses that have been found in people before.

Health officials first reported the disease in Saudi Arabia in September 2012. Through retrospective investigations, health officials later identified that the first known cases of MERS occurred in Jordan in April 2012. In that time, all cases of MERS have been linked to countries in and near the Arabian Peninsula. MERS-CoV has spread from ill people to others through close contact, such as caring for or living with an infected person. The following 26 countries have reported cases of MERS: Iran, Jordan, Kuwait, Lebanon, Oman, Qatar, Saudi Arabia, the United Arab Emirates, and Yemen (Middle East); Austria, France, Germany, Greece, Italy, Netherlands, Turkey, and the United Kingdom (UK) (Europe); Algeria, Tunisia and Egypt (Africa); China, Malaysia, Republic of Korea, the Philippines and Thailand (Asia); and the United States of America (Americas). The vast majority of these cases have so far occurred in the Kingdom of Saudi Arabia.

MERS affects lungs. Most MERS patients developed severe acute respiratory illness with symptoms of fever, cough and shortness of breath. About 3-4 out of every 10 patients reported with MERS have died. MERS can affect anyone. MERS patients have ranged in age from younger than 1 to 99 years old.

CDC continues to closely monitor the MERS situation globally and work with partners to better understand the risks of this virus, including the source, how it spreads, and how infections might be prevented. CDC recognizes the potential for MERS-CoV to spread further and cause more cases globally and in the U.S. We have provided information for travelers and are working with health departments, hospitals, and other partners to prepare for this.

A typical case of MERS includes of fever, cough, and/or shortness of breath. Pneumonia is a common finding on examination. Gastrointestinal symptoms, including diarrhoea, have also been reported. Severe illness can cause respiratory failure that requires mechanical ventilation and support in an intensive-care unit. Some patients have had organ failure, especially of the kidneys, or septic shock. The virus appears to cause more severe disease in people with weakened immune systems, older people, and those with such chronic diseases as diabetes, cancer and chronic lung disease.

It is not yet fully understood how people become infected with MERS-CoV, which is a zoonotic virus. It is believed that humans can be infected through direct or indirect contact with infected dromedary camels in the Middle East. Strains of MERS-CoV have been identified in camels in several countries, including Egypt, Oman, Qatar and Saudi Arabia.

The virus does not appear to pass easily from person to person unless there is close contact such as providing clinical care to an infected patient while not applying strict hygiene measures. This has been seen among family members, patients, and health-care workers. The majority of cases have resulted from human-to-human transmission in health care settings.

The source of the MERS-CoV is not yet fully clear. A coronavirus very similar to the one found in humans has been isolated from camels in Egypt, Oman, Qatar, and Saudi Arabia. . It is possible that 
other reservoirs exist. However, other animals, including goats, cows, sheep, water buffalo, swine, and wild birds, have been tested for MERS-CoV, but so far none have been found in these animals. These studies combined support the premise that dromedary camels are a likely source of infection in humans.

Transmission of MERS-CoV has occurred in health-care facilities in several countries, including from patients to health-care providers. It is not always possible to identify patients with MERS-CoV early or without testing because symptoms and other clinical features may be non-specific. For this reason, it is important that health-care workers apply standard precautions consistently with all patients.
Droplet precautions should be added to standard precautions when providing care to all patients with symptoms of acute respiratory infection. Contact precautions and eye protection should be added when caring for suspected or confirmed cases of MERS-CoV infection. Airborne precautions should be applied when performing aerosol-generating procedures.

\section{References}

1. Middle East respiratory syndrome coronavirus (MERSCoV). Fact sheet. Updated on: June 2015. Viewed on: 10 July 2015. Web address:

http://www.who.int/mediacentre/factsheets/mers-cov/en/

2. About MERS. Page last updated: July 6, 2015. Web address: http://www.cdc.gov/coronavirus/mers/about/index.html

Cited as: Sharif AR. Outbreak of Middle East Respiratory Syndrome (MERS): A Public Health Concern. J Curr Adv Med Res, 2015;2(2):28-29] 